\title{
Bioprosthetic aortic valve degeneration: a review from a basic science perspective
}

\author{
Tiago Velho ${ }^{1}$, Rafael Pereira ${ }^{2}$, Frederico Fernandes ${ }^{3}$, Nuno Guerra ${ }^{4}$, Ricardo Ferreira ${ }^{2}$, and \\ Angelo Nobre ${ }^{4}$ \\ ${ }^{1}$ Hospital Santa Maria \\ ${ }^{2}$ Hospital de Santa Maria \\ ${ }^{3}$ University of Algarve \\ ${ }^{4}$ University Hospital Santa Maria
}

September 21, 2020

\begin{abstract}
Background The increase in the prevalence of aortic stenosis due to an aging population has led to an increasing number of surgical aortic valve replacements. Over the past 20 years, there has been a major shift in preference from mechanical to bioprosthetic valves. However, despite efforts, there is still no "ideal" bioprosthesis. It is crucial to understand the structure, biology, and function of native heart valves to design more intelligent, strong, durable and physiological heart valve tissues. Methods A comprehensive review of the literature was performed to identify articles reporting the basic mechanisms of bioprosthetic valve dysfunction and the biology of native valve cells. Searches were run in PubMed, MEDLINEß and Google Scholar. Search terms included subject heading and keywords for the following terms: "biological heart valve dysfunction", "bioprosthesis dysfunction", "bioprosthesis degeneration" and "tissue heart valves" Results All the relevant findings are summarized in specific illustrations and tables within the appropriate subsections. Structural dysfunction is a logical and expected consequence of the chemical, mechanical and immunological processes that occur during fixation, manufacture, and implantation. Conclusion Biological prosthesis valve dysfunction is a clinically significant process. It has become a major issue considering the growing rate of bioprosthesis implantation and improved long-term patient survival. Research on the field has improved in the past few years but there is still not an ideal bioprosthesis. Understanding bioprosthetic aortic valve degeneration from a basic science perspective is a key point to improve technologic advances and specifications that lead to new generation of bioprosthesis.
\end{abstract}

\section{Bioprosthetic aortic valve degeneration: a review from a basic science perspective}

Tiago R. Velho ${ }^{1,2}$, Rafael Maniés Pereira ${ }^{1}$, Frederico Fernandes ${ }^{3}$, Nuno Carvalho Guerra ${ }^{1}$, Ricardo Ferreira ${ }^{1}$, Ângelo Nobre ${ }^{1}$

1. Cardiothoracic Surgery Department, Hospital de Santa Maria - CHLN, Lisboa, Portugal, Av. Prof. Egas Moniz, 1649-035, Lisboa, Portugal.

2. Innate Immunity and Inflammation Laboratory, Instituto Gulbenkian de Ciência, Rua da Quinta Grande n. 6, 2780-156, Oeiras, Portugal.

3. Department of Biomedical Sciences and Medicine, University of Algarve, Campus de Gambelas, 8005-139, Faro, Portugal.

Corresponding author: Tiago R. Velho, Cardiothoracic Surgery Department, Hospital de Santa Maria - CHLN, Av. Prof. Egas Moniz, 1649-035 Lisboa,Portugal; Phone: +351916461073; E-mail: tiagovelho48@hotmail.com 
Short running title: Basic biology of bioprosthesis

Conflict of Interest: None to declare.

Funding: This research did not receive any specific funding from commercial or public agencies.

Acknowledgements: None.

Word count: 5092

Abstract

Background

The increase in the prevalence of aortic stenosis due to an aging population has led to an increasing number of surgical aortic valve replacements. Over the past 20 years, there has been a major shift in preference from mechanical to bioprosthetic valves. However, despite efforts, there is still no "ideal" bioprosthesis. It is crucial to understand the structure, biology, and function of native heart valves to design more intelligent, strong, durable and physiological heart valve tissues.

\section{Methods}

A comprehensive review of the literature was performed to identify articles reporting the basic mechanisms of bioprosthetic valve dysfunction and the biology of native valve cells. Searches were run in PubMed, MEDLINER and Google Scholar. Search terms included subject heading and keywords for the following terms: "biological heart valve dysfunction", "bioprosthesis dysfunction", "bioprosthesis degeneration" and "tissue heart valves"

\section{Results}

All the relevant findings are summarized in specific illustrations and tables within the appropriate subsections. Structural dysfunction is a logical and expected consequence of the chemical, mechanical and immunological processes that occur during fixation, manufacture, and implantation.

\section{Conclusion}

Biological prosthesis valve dysfunction is a clinically significant process. It has become a major issue considering the growing rate of bioprosthesis implantation and improved long-term patient survival. Research on the field has improved in the past few years but there is still not an ideal bioprosthesis. Understanding bioprosthetic aortic valve degeneration from a basic science perspective is a key point to improve technologic advances and specifications that lead to new generation of bioprosthesis.

Key-words: bioprosthetic, valve repair/ replacement, valve dysfunction, immunology, inflammation

Bioprosthetic aortic valve degeneration: a review from a basic science perspective

\section{Introduction}

Aortic stenosis is the most common primary valve disease with indication to surgery in Europe, with an increased prevalence in the last few decades due to ageing population (1). In fact, more than 400.000 surgical aortic valve replacements (SAVR) are performed yearly worldwide (2), contributing to a significant economic and social health issue (3). It is expected that in 2050 there will be over 850.000 aortic valve replacements, worldwide (4).

There are two major types of prosthetic heart valves: mechanical or biological. Randomized clinical trials (5-7) comparing both types of prosthesis found similar generic outcomes. However, from the published studies we can draw two important conclusions: mechanical prostheses are associated with higher rates of bleeding due to anticoagulation, while bioprosthesis are associated with higher rates of reintervention due to bioprosthesis dysfunction. 
Across the world, in the past decades, there has been a considerable increase in the use of bioprosthesis over mechanical valves (8-10), with a major shift from mechanical to bioprosthetic valves in the last 20 years.. In proportion, bioprosthesis increased from $40 \%$ in the 90 s to more than $80 \%$ of all implanted prosthetic heart valves nowadays (11). This exponential increase in bioprosthetic valve implantation is likely related to an elderly patient population undergoing SAVR, a perceived improvement in valve durability, and a desire to avoid short and long-term anticoagulation (8).

Despite the continuous efforts in the past few years, still there is no "ideal" bioprosthesis. The implementation of a prosthetic heart valve always initiates several pathophysiological processes, which can lead to structural valve degeneration (SVD) and progressive clinical deterioration. Signs and symptoms of SVD depend on the type of valve, its location and the nature of the complication. There are several types of prosthetic dysfunction, ranging from structural/non-structural deterioration of the valve, thrombosis, and endocarditis (12).

For the past 50 years, glutaraldehyde (Glut) has been the most used chemical product and is currently widely used to preserve and stabilize biological prosthetic tissues. Glut is responsible for chemical crosslinking, improving the material's stability and reducing antigenicity. Bioprosthetic heart valves show several histological differences from native heart valves, being unable to remodel and repair. The manufacture process of prostheses is also crucial, especially regarding fixed configuration of the pericardial valves and the pressure used in tissue fixation.

Recently, research has brought new ideas about the inflammatory and immunological role in the dysfunction of the bioprosthesis, describing the immunological rejection and the inflammatory state also as a cause of the failure of the bioprosthesis.

In terms of durability, new generations bioprosthesis may have promising results (11). The reported durability is excellent, with rates of reintervention due to failure of the bioprosthesis of $2 \%$ to $10 \%$ in 10 years, $10 \%$ to $20 \%$ in 15 years and $40 \%$ in 20 years $(13,14)$. However, these findings do not show the true rates of deterioration of the bioprosthesis. Some studies have identified higher rates of structural deterioration, including hemodynamic changes, in up to $10 \%$ and $30 \%$ patients 5 and 10 years after surgery (11).

The significant increase in the use of aortic bioprosthesis will inevitably lead to a proportionally rising number of patients diagnosed with prosthesis dysfunction in the next decade. This should stimulate cardiac surgery centers and medical prosthesis manufacturers to understand all underlying mechanisms. This review aims to review the most debated topics on the pathophysiology of aortic bioprosthesis dysfunction, exploring the biological grounds on the chemical, mechanical and inflammatory contribution to better understand the most recent innovations in this field.

\section{Methods}

A comprehensive review of the literature was performed to identify articles reporting the basic mechanisms of bioprosthetic valve dysfunction and the biology of native valve cells. Searches were run in March 2020 in the following databases: PubMed, MEDLINER and Google Scholar. Search terms included subject heading and keywords for the following terms: "biological heart valve dysfunction", "bioprosthesis dysfunction", "bioprosthesis degeneration" and "tissue heart valves". There was no patient involvement in this study, so ethic's board approval was not required. This research received no grant or funding.

\section{Heart valves}

Natural heart valves are unique structures, adapted to allow unidirectional and nonobstructive blood flow. They are biologically dynamic structures, naturally designed to avoid regurgitation, thromboembolism, trauma to their molecular and cellular structures, or disruptive stress. To understand the pathophysiology of biological prosthetic valve's dysfunction, it is crucial to understand the structure, biology and function of native heart valves.

In the embryonic developing heart valve, epithelial-to-mesenchymal transition occurs when cells from the 
endocardium differentiate into mesenchymal cells and migrate into the cardiac jelly that forms the pre-valve cardiac cushions (15). These cushions are rich in glycosaminonoglycans (GAG), such as hyaluronic acid, and signaling molecules responsible for further development of heart valves $(1,2)$. The cardiac cushions are responsible for the complex regulation of extracellular matrix proteins, producing an intricate and functional structure (16). Although the immature heart valve produces its own extracellular matrix in utero, their development is only completed in the postnatal life $(1,3)$. Characterizing embryonic progenitors of heart valve cells and development processes is important to understand basic pathogenesis in valvular disease. Exploring cellular and molecular pathways in valvular disease will eventually allow designing more intelligent and physiological heart valve tissues.

Development of heart valves leads to a layered, structured and highly specialized complex structure of adapted cells and extracellular matrix (18). Their configuration will allow heart valves to assure its highly specified function, maintain their strength and durability despite the regular and repetitive stress and strain. Heart valves also need to have elements that assure permanent repair and remodeling. Although there are 4 different heart valves with different configurations and functions, all of them have a similar layered patter of cells.

Valve leaflets are mainly constituted by collagen type I and III, proteoglycans, GAG and elastin (19). The leaflet has three layers, each with an important microstructure (Figure 1): 1) fibrosa : the outflow surface, with circumferentially and densely aligned packed collagen fibers, to enable a load-bearing function during diastole (20); 2) spongiosa : the middle layer rich in GAG, acts as a lubricant between the two other layers (20-22); and 3) ventricularis : the inflow surface, predominantly with elastin to provide elastic proprieties $(20,21)$. The arrangement and configuration of the extracellular matrix is responsible for the changes in shape and dimensions throughout the cardiac cycle.

Two major types of cells are present in the leaflets: the valvular endothelial cells and the interstitial valvular cells. Both groups of cells synergize to maintain the normal function of the valves.

Valvular interstitical cells (VIC)

Valvular interstitial cells (VIC) represent a crucial and heterogeneous cell population through the leaflet, distributed in all layers (23). They are the most abundant cell type in the heart valves and resemble, among others, fibroblasts and smooth muscle cells. VIC represent a dynamic population responsible for the synthesis of extracellular matrix and matrix degrading enzymes. Thus, they regulate and remodel collagen and other essential components to assure the continuous valve repair (23). The activation of VICs (including the production and secretion of matrix) is regulated by mechanical stretch, local cellular signaling (e.g. interaction with other types of cells from heart valves such as valvular endothelial cells), microstructural factors, and hemodynamic environments (24). It has also been theorized that VIC contraction, in response to environment stimuli, may facilitate cell-to-cell communication and act as a role in maintaining leaflet homeostasis (25). The ability to answer to the surrounding environment makes VIC highly plastic, with at least 5 distinct phenotypes described, from a quiescent to an activated form (the 5 distinct VIC phenotypes include embryonic progenitor endothelial/ mechenchymal cells, quiescent VIC, activated VIC, postdevelopmental/ adult progenitor VIC and osteoblastic VIC) (23).

Although it may be interesting to explore all phenotypes, the quiescent and the activated forms are the most relevant in this context. VIC in adult valves are quiescent, without activity, with fibroblasts characteristics (26). The plasticity of VICs is important for development but plays also an important role in pathologic processes (19). VIC plasticity is regulated by multiple factors, such as environmental factors and host factor as age.

In the disease states, VIC progress from the quiescent fibroblast-like phenotype to a contractile form, with enhanced production and secretion of extracellular matrix, cytokines, proteases and growth factors $(9,10)$. VIC progression has direct consequences on the heart valve function, as it has been shown that VIC contraction have measurable effects on leaflet stiffness (28). When the activation persists and is prolonged, VIC can differentiate into osteoblast-like cells (the osteoblatic VIC phenotype), leading to calcific nodule formation 
and valve calcifications (29). However, activation of VIC is not a definitive process, as it can be reversiblly modulated (29).

Valvular endothelial cells (VEC)

Valvular endothelial cells (VEC) represent the cell layer that covers the leaflets, in line with the endothelium from the entire cardiovascular system. However, VEC are phenotypically different from other cardiovascular endothelial cells, probably due to the fact that they interact with VIC to maintain the integrity of valve tissues and potentially, also to mediate disease (26). They are highly specialized endothelial cells: usually endothelial cells in vascular tissues are typically elongated in the direction of blood flow, but on the leaflets they have a circumferential arrangement to support leaflet stresses and mechanical forces (30). As all endothelial cells, VEC are crucial to function as a barrier, regulating interactions between blood flow and VICs, including metabolic and inflammatory processes (31).

VEC may also exhibit differences phenotypes depending on their location on the valve leaflets (32). Endothelial cell production of nitric oxide (NO) is an important crosstalk to maintain VIC quiescent and endothelial injury has been proposed to be an initiating factor for calcified aortic valve disease (33). VEC are also responsible for the non-thrombogenic proprieties of the leaflets, playing an important immune and inflammatory role (34). VEC are also capable of responding to mechanical stimuli, with a flow-mediating mechanotransduction process being responsible for the activation of protective or pathological pathways (35).

\section{Heart Valves as a Functional System}

Understand the molecular and cellular components of the heart valves will allow us to understand the biomechanical features of the valves.

The aortic valve is the most frequently diseased and studied valve, and represents the perfect paradigm to represent the complex and highly specialized extracellular matrix configuration of heart valves. During diastole, the aortic valve leaflets stretch to avoid blood backflow. The change in its configuration is predominantly dependent on collagen, with directional realignment and crimping of the fibers. The collagen orientation determines tissue compliance to tensile stress. This complex fiber architecture is very sensitive to pressure, and very low pressures at the beginning of the cardiac cycle are capable of inducing collagen fibers arrangements, with loss of the fibrosa corrugations and collagen crimps (15-18). Mechanical functions of collagen also include limitation of cuspal stretching to avoid proplapse (achieved mainly by the strained and aligned collagen in the fibrosa) (23).

During systolic valve opening, the tissue becomes relaxed, with the elastin from the ventricularis layer recoiling to make the cusp retracted again $(20,26)$. In this phase of the cardiac cycle, fibers have a random directional distribution and crimps of collagen fibrils are restored (23).

All these continuous and coordinated alterations in valve configuration depend on the quality and quantity of its components, such us collagen, elastin and GAG. Indeed, they are the major determinants of the functional mechanisms and long-term durability of the valve. It has already been showed that the cell source used in bioprosthesis is important for long-term durability since the lack of cells in bioprosthesis has been shown to be the main source of failure for implants (26). That is the reason why all the previously described components are essential to maintain valve homeostasis. Thus, cell source and function are an important component of heart valve tissues, as they depend on the viability and function of active valve cells (23).

\section{Bioprosthesis Dysfunction}

Biological heart valves consist of fixed or decellularized human or animal (usually porcine or bovine) tissue, attached or not to a stent (without a stent in the stentless prosthesis) and a sewing ring (without sewing ring in the sutureless prosthesis). Structural dysfunction is a logical and expected consequence of the chemical, mechanical and immunological processes that occur during fixation, fabrication and implantation.

Beside all the technological development in the past decades, bioprosthesis only replicate native heart valve structure. In bioprosthesis, 1) the cusps are constituted by subendothelial connective tissue; 2) the cusps are 
locked in a static geometry and 3) have nonviable cells due to the fixation and crosslinking processes (21, 22). They lack the functional capacities of heart valves and the preservation techniques severely decrease functional activity of the natural matrix (17). Thus, they are not able to maintain the normal remodeling processes.

Over the past 50 years, glutaraldehyde (Glut) has been the most used chemical and is nowadays widely used to preserve and stabilize biological prosthetic tissues. Glut is an aldehyde with fixative and preservative functions (43), firstly introduced in 1963 by Sabatini et al as a fixative for electronic microscopy. Glut is responsible for the chemical crosslinking (creation of covalent chemical bonds to stabilize tissues and terminate any ongoing biochemical reactions), enhancing material stability and reducing antigenicity (29, 30). However, Glut is also partly responsible for prosthesis dysfunction, directly because of its toxicity and indirectly through the processes described ahead.

Valve degeneration is a multifactorial process, including chemical, mechanical and immunological factors (Figure 2). The contribution of each mechanism remains poorly understood, but represents an active and attractive field of investigation. The common endpoint of all these processes is calcification and degradation, culminating in the failure of the structure with bioprosthetic dysfunction.

Chemical contribution

Glut crosslinking make tissues biocompatible and nontrombogenic, while maintaining anatomic integrity, leaflet strength and flexibility (45). Its preference among aldehydes is related to its availability, low price, quick action and capacity to react with a large number of amino acids (45).

Glut is responsible for the effective crosslinking of collagen, the most common structural component of the valves. It forms covalent bonds by the formation of Schiff bases (reaction of an aldehyde group with an amino group of lysine or hydroxylysine) and/ or by aldol condensation between two adjacent aldehydes (46). Crosslinking increases tissues durability, reducing resistance to proteolysis of the cross-linked proteins. However, after Glut fixation residual aldehydes remain expressed on the tissue surface and may act as calcification locations.

Although its action is essential for valve preservation and to eliminate cellular components to reduce tissue immunogenicity, the induced chemical reactions are probably the most important part of bioprosthetic valve degeneration. After Glut fixation, valvular interstitial cells lose their viability (42). However, some studies have showed that Glut retains many of the viscoelastic proprieties of the collagen (45), with haemodynamic proprieties of the prostheses similar to those of living tissues (45).

Additionally, as part of the fixation and fabrication process, the cellular content of the tissues is modified, with loss of endothelial cells, loss of interstitial cell viability and interstitial cell degeneration (41). Indeed, bioprosthetic heart valves show several histological differences from native heart valves, with flattening of the cuspal corrugations, loss of the endothelium or mesothelium surfaces, disruption of interstitial cells and loss of GAG (42). With the loss of interstitial cells viability, the mechanical proprieties and durability of the valve depends primarily on the quality of the collagen and the remaining viscoelastic proprieties are not enough to avoid valve tissue degeneration.

Schoen et al described the calcification process as having two phases: nucleation (or initiation) and propagation (41). One important change to initiate calcification is the abnormal extrusion of calcium ions from the nonviable cells. Cross-linking to proteins of the cellular membrane alters their proprieties, resulting in a different permeability in the non-viable cells. Additionally, there is a the reduction of the functional transmembrane ion pumps and an increased permeability to calcium ions that contribute to the onset of calcifications (47). Usually calcium concentration is 1000 to 10.000 times lower in the cytoplasm due to the healthy ion pumps that carry calcium out of the cells. With deregulated calcium levels inside the cells, cellular membranes and other intracellular structures bind calcium and serve as nucleators for calcifications. Indeed, calcification seems to start predominantly at the cell membranes and other intracellular structures rich in phospholipids, while the loss of proteoglycans may enhance this phenomenon by removing calcificati- 
on inhibitors (48). Glut also reacts directly with intracellular structures, predisposing to calcification in the presence of high intracellular calcium levels (49). The debris of interstitial cells that remain in valve tissue also serves as initiation sites for calcification.

Collagen and elastic fibers can also serve as nucleation sites, independent of cellular components. One important difference is that calcification of collagen requires cross-linking alterations, while calcification of elastin occur independently of cross-linking (50).

After the nucleation, calcification is influenced by all the metabolic and pathologic changes in calcium and phosphorus metabolism, with calcium-enriched crystals growing to eventually culminate in prosthesis malfunction (propagation phase).

Another important characteristic of native heart valves is their remodeling and reparation capacity. In biological prosthesis the fixed and nonviable tissue is incapable to maintain the ongoing repair, and every damage do the extracellular matrix is cumulative. Moreover, endothelial is denuded or absent and adjacent smooth muscle cells might proliferate and migrate freely to the non-endothelized valve surface (51), also contributing to valve dysfunction.

The dynamic role of native valve cells' and their importance for the durability of bioprosthesis is now recognized, and new strategies of repopulation and regeneration have been proposed to minimize the problem. Repopulation defines the process of using a clean connective tissue matrix valve, repopulated with the recipient cells', before or after prosthesis implantation (52). A completely "self-populated" prosthesis would maintain tissues invisible, avoiding host reaction to the graft. Although this technology reached clinical practice, the results were not as good as expected. Regeneration involves the implantation of a remodeling matrix with the proteins and cells of the recipient that can resemble the dynamic changes of native heart valves (52), and remains in the pre-clinical development.

Storage is another important issue regarding bioprosthetic valve dysfunction. Actually, several bioprosthesis are stored in liquids containing aldehydes, which are toxic and a source for calcification, as previously described in this chapter. Regardless fixation and production procedures with a reduced aldehyde content, tissues are exposed once again to deleterious free aldehydes when they are stored in aldehyde enriched solutions. Even pre-implant rinsing does not guarantee complete removal of toxic aldehydes with such storage solutions, and new technologies regarding storage are also an active field of research.

\section{Mechanical}

In the past heart valve dysfunction was assumed as a degenerative and passive deposition of calcium crystals. However, it has been established that biological prosthetic valve dysfunction is a very dynamic and progressive process, including noncalcific deterioration with important mechanical and environmental contributions.

As part of the fabrication model of bioprosthesis, the structure is fixed in a static geometry. The collagenous network is locked into a single configuration of one phase of the cardiac cycle, inhibiting the normal extracellular matrix readjustments of the valve during the cardiac cycle $(39,40)$. The collagen crimps and corrugations are preloaded in a defined configuration similar to that of a closed valve (55). Thereby, functional and normal cardiac cycle stresses have to be absorbed by the noncompliant and fixed collagen fibers (55). Having a fixed configuration will produce damage in pericardial valves during closure (in porcine valves mostly during opening but also during closing), inducing repetitive accumulation of mechanical stress with increased tissue fatigue $(39,40)$. Leaflets should display anisotropic proprieties, especially regarding strain in the circumferential and radial directions. It has been established that non-physiological strain leads to pathologic processes by deregulation of inflammation and remodeling, leading to calcification (18).

The pressure used during fixation is also important for the mechanical changes induced in the tissues, as it determines the stress-strain relation of the tissue strips (58). Low pressures have better mechanical proprieties and most of the bioprosthesis used nowadays, such as Edwards Lifesciences@ Magna Perimount

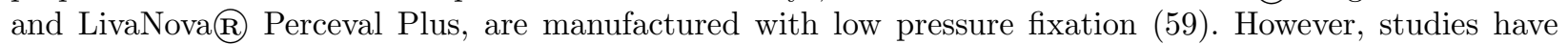
shown that as low as 2-4 mmHg are sufficient to induce significant changes in collagen compliance (55), so 
zero-pressure methods have been studied. Although in theory zero-pressure would offer a new approach to reduce biological prosthetic dysfunction, the technique has not achieved clinically significant results (60).

Although Glut-related dysfunction is more associated with calcification, it also induces mechanical alterations to the valve tissue. It has been shown that glut alters the stress-strain curves of strips of bovine and porcine valve tissues (58). During the fixation process there is also a considerable loss and incomplete stabilization of the GAGs (61), which are responsible for the viscoelasticity and accommodation of the cuspal layers. GAGs have an essential role in absorbing compressive loads, modulating shear stress and avoiding tissue buckling, and they may be important in mechanical abnormalities that lead to valve dysfunction.

The chemical and mechanical changes are possible synergetic, since changes in tissue configuration can induce stress and fatigue (especially in flexion lines of the cusps) that can expose and disrupt collagen, initiating the calcification process. On the other hand, calcifications can also induce structural changes and stiffness that will eventually lead to more mechanical changes and damage.

Immunological

Recently, research has brought new insights on the inflammatory and immunological role in bioprosthesis dysfunction, describing immune rejection also as a cause for bioprosthesis failure $(43,44)$.

In all biological processes, immune responses have several grades, from a physiological to pathologic state. Even in the setting of a bioprosthesis, inflammation can be divided into several types: 1) the postsurgical normal wound healing; 2) nonspecific innate inflammatory reaction to a new foreign body; and 3) immune-mediated rejection and/ or inflammation (64-66). We will focus on point 2 and 3, since the normal postsurgical wound healing is not in the scope of this review.

Despite all the fixation and processing procedures, Glut decreases but not entirely eliminates the antigenicity of tissue valves (67). Collagen matrix (with cell debris and necrotic products) elicits a strong nonspecific inflammatory response, including infiltration by macrophages and eosinophils, followed by a lymphocyte $\mathrm{T}$ response (68). Persistent antigenicity of bioprosthesis has been shown to continually stimulate graft-specific adaptive immune reactions with important biomaterial dysfunction (68). The dysfunctional endothelial layer may also contribute to maintain the inflammatory status, probably through the reduced nitric oxide production and increased generation of reactive oxygen species and inflammatory cytokines (69).

Glut itself also causes some degree of inflammation (70). Additionally, inflammation and calcification are also linked. It has been shown that calcification is associated with the amount of inflammation, as lymphocytes and macrophages produce osteopontin (an important cytokine in the calcification process) (70). Inflammatory and fibroblast signaling contribute to a pro-osteogenic environment (with the activation of quiescent VIC to the osteogenic VIC phenotype) and remodeling process, predisposing dystrophic calcification (71).

Host cardiovascular risk factors may also contribute to the inflammatory environment. Studies have demonstrated that some risk factor such dyslipidemia, diabetes or metabolic syndrome may modulate bioprosthesis degeneration through inflammation $(53,54)$. Analyzes from the explanted prosthesis have also revealed that explants are usually infiltrated by oxidized low-density lipoproteins (LDL), beside inflammatory cells (74). Indeed, patients with SVD have a tendency of higher triglyceride levels and high levels of small, dense LDL, which are associated with prosthesis dysfunction (74). Moreover, Mahmut et al. have shown that lipoproteinassociated phospholipase A2 (Lp-PLA2), an enzyme that produces pro-inflammatory substances from LDL, is an independent predictor of SVD (75).

Another important factor that has recently been associated with bioprosthetic valve dysfunction is Alphagal. Alpha-gal (galactose-alpha-1,3-galactose) is a carbohydrate found in most mammalian membranes, but not in humans. Humans normally display anti-gal antibodies due to antigenic stimulation, representing an important barrier to xenotransplantation (76). Yet, alpha-gal epitopes are present in bioprosthesis, even in decellularized tissues. It has been shown that the implantation of bioprosthesis induce a specific immune reaction to the alpha-gal antigen, with the production of anti-alpha-gal antibodies (76). The interaction between the circulation anti-alpha gal antibodies and calcification of bioprosthesis has been established 
$(58,59)$, but the contribution to long-term dysfunction is not yet completely understood. However, basic research continues and recent studies have shown that engineered pericardial tissue from alpha-gal-deficient pigs calcify less in animal models (78). Tissue valve investigation continues in order to design new tissues with less alpha-gal epitopes, and a genetically modified pig with no expression of alpha-gal has already been generated, but more studies are needed (79).

\section{New generation of aortic bioprosthesis: technology specifications}

One important point in tissue valve engineering is the ability to develop new biological prosthesis with less static configurations and more biologically active tissues. So far, recent technologies have achieved better fixation and storage procedures (Table 1), but all of them fail to mimic biological activity of heart valve cells. Recently, two new bioprosthesis (Inspiris $\AA$ from Edwards Lifesciences@ and Perceval Plus@ from Livanova@) with two novel tissue treatments (Resiliaß and FREEß, respectively) have been launched expecting less calcification and improved tissue durability.

Perceval Plus@ is a bovine pericardial heart valve with a novel tissue treatment to reduce calcification: the FREE (R treatment. FREE treatment uses an alcohol mixture for phospholipids removal, combined with a post-sterilization amino-acid treatment for the neutralization of unbound aldehydes, and final storage with an aldehyde-free solution (80). FREE-treated tissues have a reduced content of phospholipids up to $96 \%$, comparing to Glut-treated tissues, combined with a significant improvement in the removal and neutralization of unbound Glut (80). Tissues have less propensity to mineralization while maintaining the same mechanical and biochemical performance and stability of the conventional Glut-treated prosthesis (80). In theory, FREE treatment is an effective strategy to reduce bioprosthesis dysfunction. However, long-term outcomes in humans still unknown.

Resilia $(\mathbb{B}$ is also a new tissue preservation technology, that uses stable functional group capping, ethylene oxide sterilization, and preservation by glycerolization (81). This innovative technology does not avoid Glut use but reduces phospholipids content and residual chemicals from the valve tissues. There are also differences considering the storage of the tissues, using dry storage, which reduces the contact of the prosthesis with aldehyde-enriched solutions.

Despite all these new and exciting tissue treatments to prevent calcification, we should keep in mind that it will take longer to have the 15-20 year outcomes we now have for conventional Glut-treated tissues. Moreover, all these strategies are based on the reduction of free aldehyde groups and phospholipids content, reducing the chemical effects of Glut. As previously discussed here, chemical changes are just part of the complex process of biological prosthetic valves dysfunction, and new strategies to address mechanical and immunological changes in valve tissues must be addressed.

\section{Conclusion}

Biological prosthesis valve dysfunction is a clinically significant process. It has become a major issue and a hot topic considering the growing rate of bioprosthesis implantation and improved long-term patient survival.

Although biological prosthesis has important advantages over mechanical, durability is the major limitation of their implantation. The design and development of new biological bioprosthesis must reproduce the structure, and, more important, the biology of native heart valves. Understanding the complex biologically functional and dynamic system of the heart valves will elucidate how bioprosthesis can match their natural behavior. Every surgeon should be aware of their biological complexity to understand and discuss new tissue valve technologies, to provide patients heart valves with improved durability and better performance.

Although biological heart valves have been used for more than 50 years, bioprosthesis dysfunction remains a challenging and intriguing field, with an enormous quantity of work and research ahead in each of its subjects. SVD remains an intriguing field because the pathophysiology of SVD is not yet completely known. Only in the past few years, studies have unveiled new biological pathways. And that is the reason why basic research on the pathophysiology of SVD remains crucial. We will only achieve new and efficient tissue development if we deepen our knowledge of the biological processes of native heart valves. Fortunately, research on tissue 
heart valves is now wide and covers several different fields, such as 1) the effects of Glut-fixation, 2) non-Glut fixation, 3) mechanisms of calcification and non-calcification dysfunction, 4) anticalcification approaches, 5) biology of the valvular cells and 6) tissue-engineered valves (41).

Concluding, biological heart prosthesis dysfunction is a complex and multifactorial process, with biological, chemical, mechanical and immunological factors. Chemical changes caused by Glut, and consequent calcification, have been the main target of tissue valve development. However, as pathophysiology has been explored, with more detailed knowledge on cell structure and function and on the inflammation associated with bioprosthetic valve implantation, new questions arise. SVD remains a challenging field of research and novel interventions and developments need to focus on strategies that target the cell and immune events responsible for degeneration and rejection of the tissues. Moreover, new tissues must keep some of the biological proprieties of native heart valves, since valve changes in conformation during the cardiac cycle and regeneration processes are two essential but many times forgotten aspects of SVD.

\section{References}

1. Baumgartner H, Falk V, Bax JJ, De Bonis M, Hamm C, Holm PJ, et al. 2017 ESC/EACTS Guidelines for the management of valvular heart disease. Eur Heart J. 2017 Sep;38(36):2739-91.

2. Nitsche C, Kammerlander AA, Knechtelsdorfer K, Kraiger JA, Goliasch G, Dona C, et al. Determinants of Bioprosthetic Aortic Valve Degeneration. JACC Cardiovasc Imaging [Internet]. 2020 Feb;13(2):345-53. Available from: https://linkinghub.elsevier.com/retrieve/pii/S1936878X19301652

3. Moore M, Chen J, Mallow PJ, Rizzo JA. The direct health-care burden of valvular heart disease: Evidence from US national survey data. Clin Outcomes Res. 2016;8:613-27.

4. Yacoub MH, Takkenberg JJM. Will heart valve tissue engineering change the world? Nat Clin Pract Cardiovasc Med. 2005;2(2):60-1.

5. Stassano P, Di Tommaso L, Monaco M, Iorio F, Pepino P, Spampinato N, et al. Aortic Valve Replacement. A Prospective Randomized Evaluation of Mechanical Versus Biological Valves in Patients Ages 55 to 70 Years. J Am Coll Cardiol. 2009;54(20):1862-8.

6. Hammermeister K, Sethi GK, Henderson WG, Grover FL, Oprian C, Rahimtoola SH. Outcomes 15 years after valve replacement with a mechanical versus a bioprosthetic valve: Final report of the Veterans Affairs randomized trial. J Am Coll Cardiol. 2000;36(4):1152-8.

7. Oxenham H, Bloomfield P, Wheatley DJ, Lee RJ, Cunningham J, Prescott RJ, et al. Twenty year comparison of a Bjork-Shiley mechanical heart valve with porcine bioprostheses. Heart. 2003;89(7):715-21.

8. Degeneration TV. Aortic Bioprosthetic Valve Durability. 2017;70(8).

9. Bartus K, Sadowski J, Litwinowicz R, Filip G, Jasinski M, Deja M, et al. Changing trends in aortic valve procedures over the past ten years - From mechanical prosthesis via stented bioprosthesis to TAVI procedures - Analysis of 50,846 aortic valve cases based on a polish national cardiac surgery database. J Thorac Dis. 2019;11(6):2340-9.

10. Dunning J, Gao H, Chambers J, Moat N, Murphy G, Pagano D, et al. Aortic valve surgery: Marked increases in volume and significant decreases in mechanical valve use - An analysis of 41,227 patients over 5 years from the Society for Cardiothoracic Surgery in Great Britain and Ireland National database. J Thorac Cardiovasc Surg. 2011;142(4):776-782.e3.

11. Salaun E, Clavel MA, Rodés-Cabau J, Pibarot P. Bioprosthetic aortic valve durability in the era of transcatheter aortic valve implantation. Heart. 2018;104(16):1323-32.

12. Capodanno D, Petronio AS, Prendergast B, Eltchaninoff H, Vahanian A, Modine T, et al. Standardized definitions of structural deterioration and valve failure in assessing long-term durability of transcatheter and 
surgical aortic bioprosthetic valves: A consensus statement from the European Association of Percutaneous Cardiovascular Interven. Eur J Cardio-thoracic Surg. 2017;52(3):408-17.

13. Johnston DR, Soltesz EG, Vakil N, Rajeswaran J, Roselli EE, Sabik JF, et al. Long-term durability of bioprosthetic aortic valves: Implications from 12,569 implants. Ann Thorac Surg. 2015;99(4):1239-47.

14. Bourguignon T, Bouquiaux-Stablo AL, Candolfi P, Mirza A, Loardi C, May MA, et al. Very long-term outcomes of the carpentier-edwards perimount valve in aortic position. Ann Thorac Surg. 2015;99(3):831-7.

15. Sewell-Loftin MK, Young WC, Khademhosseini A, Merryman WD. EMT-inducing biomaterials for heart valve engineering. J Cardiovasc Transl Res. 2012;4(5):658-71.

16. Armstrong E, Bischoff J. Heart Valve Development: Endothelial Cell Signaling and Differentiation Ehrin. Circ Res. 2004;95(5):458-70.

17. Blum KM, Drews JD, Breuer CK. Tissue-Engineered Heart Valves: A Call for Mechanistic Studies. Tissue Eng - Part B Rev. 2018;24(3):240-53.

18. Arjunon S, Rathan S, Jo H, Yoganathan AP. Aortic Valve: Mechanical Environment and Mechanobiology. Ann Biomed Eng. 2017;41(7):1331-46.

19. Chester AH, Taylor PM. Molecular and functional characteristics of heart-valve interstitial cells. Philos Trans R Soc B Biol Sci. 2007;362(1484):1437-43.

20. Vesely I, Noseworthy R. Micromechanics of the fibrosa and the ventricularis in aortic valve leaflets. J Biomech. 1992;25(1).

21. Vesely I. The role of elastin in aortic valve mechanics. J Biomech. 1997;31(2):115-23.

22. Christie GW. Anatomy of aortic heart valve leaflets: The influence of glutaraldehyde fixation on function. Eur J Cardio-thoracic Surg. 1992;6:S25-33.

23. Schoen FJ. Evolving concepts of cardiac valve dynamics: The continuum of development, functional structure, pathobiology, and tissue engineering. Circulation. 2008;118(18):1864-80.

24. Nachlas ALY, Li S, Davis ME. Developing a Clinically Relevant Tissue Engineered Heart Valve-A Review of Current Approaches. Adv Healthc Mater. 2017;6(24):1-30.

25. Li RL, Russ J, Paschalides C, Ferrari G, Waisman H, Kysar JW, et al. Mechanical Considerations for Polymeric Heart Valve Development : Biomechanics, Materials, Design and Manufacturing. Biomaterials. $2019 ; 119493$.

26. Sacks MS, Schoen FJ, Mayer JE. Bioengineering Challenges for Heart Valve Tissue Engineering. :289314.

27. Liu AC, Joag VR, Gotlieb AI. The emerging role of valve interstitial cell phenotypes in regulating heart valve pathobiology. Am J Pathol. 2007;171(5):1407-18.

28. El-Hamamsy I, Balachandran K, Yacoub MH, Stevens LM, Sarathchandra P, Taylor PM, et al. Endothelium-Dependent Regulation of the Mechanical Properties of Aortic Valve Cusps. J Am Coll Cardiol. $2009 ; 53(16): 1448-55$.

29. Walker GA, Masters KS, Shah DN, Anseth KS, Leinwand LA. Valvular myofibroblast activation by transforming growth factor- $\beta$ : Implications for pathological extracellular matrix remodeling in heart valve disease. Circ Res. 2004;95(3):253-60.

30. Deck JD. Endothelial cell orientation on aortic valve leaflets. Cardiovasc Res. 1986;20(10):760-7.

31. Leask RL, Jain N, Butany J. Endothelium and valvular diseases of the heart. Microsc Res Tech. $2003 ; 60(2): 129-37$. 
32. Butcher JT, Nerem RM. Valvular endothelial cells and the mechanoregulation of valvular pathology. Philos Trans R Soc B Biol Sci. 2007;362(1484):1445-57.

33. Bosse K, Hans CP, Zhao N, Koenig SN, Huang N, Guggilam A, et al. Endothelial nitric oxide signaling regulates Notch1 in aortic valve disease. J Mol Cell Cardiol. 2013;60(1):27-35.

34. Cheung DY, Duan B, Butcher JT. Current progress in tissue engineering of heart valves: Multiscale problems, multiscale solutions. Expert Opin Biol Ther. 2015;15(8):1155-72.

35. Davies PF. Multiple signaling pathways in flow-mediated endothelial mechanotransduction: PYK-ing the right location. Arterioscler Thromb Vasc Biol. 2002;22(11):1755-7.

36. Hilbert SL, Barrick MK, Ferrans VJ. Porcine aortic valve bioprostheses: A morphologic comparison of the effects of fixation pressure. J Biomed Mater Res. 1990;24(6):773-87.

37. Sacks MS, Smith DB, Hiester ED. The aortic valve microstructure: Effects of transvalvular pressure. J Biomed Mater Res. 1998;41(1):131-41.

38. Lo D, Vesely I. Biaxial strain distributions in expanded porcine bioprosthetic valves. J Heart Valve Dis. 2002;11(5):688-95.

39. Vesely I. Reconstruction of loads in the fibrosa and ventricularis of porcine aortic valves. ASAIO J. $1996 ; 42(5)$.

40. Scott M, Vesely I. Morphology of porcine aortic valve cusp elastin. J Hear Valve Dis. 1996;5(5):464-71.

41. Schoen FJ, Levy RJ. Founder' s Award, 25th Annual Meeting of the Society for Biomaterials , Providence, RI , April 28 - May 2, 1999 Tissue Heart Valves : Current Challenges and Future Research Perspectives. 1999;

42. Ferrans VJ, Spray TL, Billingham ME, Roberts WC. Structural changes in glutaraldehyde-treated porcine heterografts used as substitute cardiac valves. Transmission and scanning electron microscopic observations in 12 patients. Am J Cardiol. 1978;41(7):1159-84.

43. Golomb G, Schoen F, Smith M, Linden J, Dixon M, Levy R. The role of glutaraldehyde-induced cross-links in calcification of bovine pericardium used in cardiac valve bioprostheses. Am J Pathol. 1987;127(1):122-30.

44. Southern L, Hughes H, Lawford P, Clench M, Manning N. Glutaraldehyde-induced cross-links: a study of model compounds and commercial bioprosthetic valves. J Hear Valve Dis. 2000;9(2):241-8.

45. Jayakrishnan A, Jameela SR. Glutaraldehyde as a fixative in bioprostheses and drug delivery matrices. Biomaterials. 1996;17(5):471-84.

46. Grant M, Prockop D. The biosynthesis of collagen. N Engl J Med. 1972;286(4):194-9.

47. Chen W, Schoen FJ, Levy RJ. Mechanism of efficacy of 2-amino oleic acid for inhibition of calcification of glutaraldehyde-pretreated porcine bioprosthetic heart valves. Circulation. 1994;90(1):323-9.

48. Valente M, Bortolotti U, Thiene G. Ultrastructural Substrates of Dystrophic Calc fi cation in Porcine Bioprosthetic Valve Failure. Am J Pathol. 1985;119(1):12-21.

49. Schoen FJ, Tsao JW, Levy RJ. Calcification of bovine pericardium used in cardiac valve bioprostheses: Implications for the mechanisms of bioprosthetic tissue mineralization. Am J Pathol. 1986;123(1):134-45.

50. Vyavahare N, Ogle M, Schoen FJ, Levy RJ. Mechanisms of elastin calcification and its prevention with aluminum chloride pretreatment. Annu Int Conf IEEE Eng Med Biol - Proc. 1999;2(3):760.

51. Lopez-Moya M, Melgar-Lesmes P, Kolandaivelu K, Hernandez J, Edelman E, Balcells M. Optimizing glutaraldehyde-fixed tissue heart valves with chondroitin sulfate hydrogel for endothelialization and shield against deterioration. Biomacromolecules. 2018;19(4):1234-44. 
52. Vesely I. Heart valve tissue engineering. Circ Res. 2005;97(8):743-55.

53. Vesely I, Boughner D, Song T. Tissue Buckling as a Mechanism of Bioprosthetic Valve Failure. Ann Thorac Surg. 1988 Sep;46(3):302-8.

54. Fisher J, Davies GA. Buckling in bioprosthetic valves. Ann Thorac Surg. 1989 Jul;48(1):147-8.

55. Schoen F. Aortic valve structure-function correlations: role of elastic fibers no longer a stretch of the imagination. J Hear Valve Dis. 1997;6(1):1-6.

56. Broom ND. An "in vitro" study of mechanical fatigue in glutaraldehyde-treated porcine aortic valve tissue. Biomaterials. 1980;1(1):3-8.

57. Gabbay S, Kadam P, Factor S, Cheung T. Do heart valve bioprostheses degenerate for metabolic or mechanical reasons? J Thorac Cardiovasc Surg. 1988;95(2):208-15.

58. Broom ND, Thomson FJ. Influence of fixation conditions on the performance of glutaraldehyde-treated porcine aortic valves: towards a more scientific basis. Thorax. 1979;34(2):166-76.

59. Sellke F, Del Nido P, Swanson S. Sabiston and Spencer Surgery of the Chest. 9th ed. Elsevier; 2015.

60. Revanna P, Fisher J, Watterson K. The influence of free hand suturing technique and zero pressure fixation on the hydrodynamic function of aortic root and aortic valve leaflets. Eur J Cardio-Thoracic Surg. 1997 Feb;11(2):280-6.

61. Shah SR, Vyavahare NR. The Effect of Glycosaminoglycan Stabilization on Tissue Buckling in Bioprosthetic Heart Valves. Biomaterials. 2008;29(11):1645-53.

62. Villa ML, De Biasi S, Pilotto F. Residual Heteroantigenicity of Glutaraldehyde-Treated Porcine Cardiac Valves. Tissue Antigens. 1980;16(1):62-9.

63. Human P, Zilla P. The possible role of immune responses in bioprosthetic heart valve failure. J Hear Valve Dis. 2001;10(4):460-6.

64. Hooper D, Hawkins J, Fuller T, Profaizer T, Shaddy R. Panel-reactive antibodies late after allograft implantation in children. Ann Thorac Surg. 2005;79(2):641-4.

65. Ketchedjian A, Kreuger P, Lukoff H, Robinson E, Linthurst-Jones A, Crouch K, et al. Ovine panel reactive antibody assay of HLA responsivity to allograft bioengineered vascular scaffolds. J Thorac Cardiovasc Surg. 2005;129(1):159-66.

66. Dignan R, O'Brien M, Hogan P, Thornton A, Fowler K, Byrne D, et al. Aortic valve allograft structural deterioration is associated with a subset of antibodies to human leukocyte antigens. J Hear Valve Dis. 2003;12(3):382-90.

67. Pibarot P, Dumesnil JG. Prosthetic heart valves: Selection of the optimal prosthesis and long-term management. Circulation. 2009;119(7):1034-48.

68. Simon P, Kasimir MT, Seebacher G, Weigel G, Ullrich R, Salzer-Muhar U, et al. Early failure of the tissue engineered porcine heart valve SYNERGRAFT ${ }^{\mathrm{TM}}$ in pediatric patients. Eur J Cardio-thoracic Surg. 2003;23(6):1002-6.

69. Li H, Forstermann U. Nitric oxide in the pathogenesis of cardiac disease. J Pathol. 2000;190:144-254.

70. Manji RA, Zhu LF, Nijjar NK, Rayner DC, Korbutt GS, Churchill TA, et al. Glutaraldehyde-fixed bioprosthetic heart valve conduits calcify and fail from xenograft rejection. Circulation. 2006;114(4):318-27.

71. Vattikuti R, Towler DA. Osteogenic regulation of vascular calcification: An early perspective. Am J Physiol - Endocrinol Metab. 2004;286(5 49-5):686-96. 
72. Briand M, Pibarot P, Després JP, Voisine P, Dumesnil JG, Dagenais F, et al. Metabolic syndrome is associated with faster degeneration of bioprosthetic valves. Circulation. 2006;114(SUPPL. 1).

73. O'Brien KD. Do bioprosthetic aortic valves deteriorate more rapidly in patients with the metabolic syndrome?: Commentary. Nat Clin Pract Cardiovasc Med. 2007;4(4):192-3.

74. Shetty R, Côté N. Elevated Proportion of Small, Dense Low-Density Lipoprotein Particles and Lower Adiponectin Blood Levels Predict Early Structural Valve Degeneration of Bioprostheses. 2012;20-6.

75. Mahmut A, Mahjoub H, Boulanger MC, Fournier D, Després JP, Pibarot P, et al. Lp-PLA2 is associated with structural valve degeneration of bioprostheses. Eur J Clin Invest. 2014;44(2):136-45.

76. McGregor C, Kogelberg H, Vlasin M, Byrne G. Gal-knockout bioprostheses exhibit less immune stimulation compared to standard biological heart valves. J Hear Valve Dis. 2013;22(3):383-90.

77. McGregor CGA, Carpentier A, Lila N, Logan JS, Byrne GW. Cardiac xenotransplantation technology provides materials for improved bioprosthetic heart valves. J Thorac Cardiovasc Surg. 2011;141(1):269-75.

78. Lila N, McGregor CGA, Carpentier S, Rancic J, Byrne GW, Carpentier A. Gal knockout pig pericardium: New source of material for heart valve bioprostheses. J Hear Lung Transplant. 2010;29(5):538-43.

79. Oveissi F, Naficy S, Lee A, Winlaw DS, Dehghani F. Materials and manufacturing perspectives in engineering heart valves: a review. Mater Today Bio. 2020;5(September 2019).

80. Meuris B, De Praetere H, Strasly M, Trabucco P, Lai JC, Verbrugghe P, et al. A novel tissue treatment to reduce mineralization of bovine pericardial heart valves. J Thorac Cardiovasc Surg. 2018;156(1):197-206.

81. Flameng W, Hermans H, Verbeken E, Meuris B. A randomized assessment of an advanced tissue preservation technology in the juvenile sheep model. J Thorac Cardiovasc Surg. 2015;149(1):340-5.

82. Vyavahare NR, Hirsch D, Lerner E, Baskin JZ, Zand R, Schoen FJ, et al. Prevention of calcification of glutaraldehyde-crosslinked porcine aortic cusps by ethanol preincubation: Mechanistic studies of protein structure and water-biomaterial relationships. J Biomed Mater Res. 1998;40(4):577-85.

83. Vyavahare N, Hirsch D, Lerner E, Baskin JZ, Schoen FJ, Bianco R, et al. Prevention of Bioprosthetic Heart Valve Calcification by Ethanol Preincubation. Circulation. 1997 Jan 21;95(2):479-88.

84. Perez de Arenaza D, Lees B, Flather M, Nugara F, Husebye T, Jasinski M, et al. Randomized Comparison of Stentless Versus Stented Valves for Aortic Stenosis. Circulation. 2005 Oct 25;112(17):2696-702.

85. Repossini A, Rambaldini M, Lucchetti V, Da Col U, Cesari F, Mignosa C, et al. Early clinical and haemodynamic results after aortic valve replacement with the Freedom SOLO bioprosthesis (experience of Italian multicenter study). Eur J Cardio-Thoracic Surg. 2012 May 1;41(5):1104-10.

86. Stacchino C, Bona G, Bonetti F, Rinaldi S, Della Ciana L, Grignani A. Detoxification Process for Glutaraldehyde-Treated Bovine Pericardium: Biological, Chemical and Mechanical Characterization. J Hear Valve Dis. 1998;7(2):190-4.

87. David TE, Armstrong S, Maganti M. Hancock II Bioprosthesis for Aortic Valve Replacement: The Gold Standard of Bioprosthetic Valves Durability? Ann Thorac Surg. 2010 Sep;90(3):775-81.

88. Bortolotti U, Milano A, Mazzucco A, Guerra F, Magni A, Santini F, et al. The Hancock II porcine bioprosthesis. A preliminary report. J Thorac Cardiovasc Surg. 1989 Mar;97(3):415-20.

89. Arbustini E, Jones M, Moses RD, Eidbo EE, Carroll RJ, Ferrans VJ. Modification by the hancock T6 process of calcification of bioprosthetic cardiac valves implanted in sheep. Am J Cardiol. 1984 May;53(9):1388-96.

90. Rieß F-C, Fradet G, Lavoie A, Legget M. Long-Term Outcomes of the Mosaic Bioprosthesis. Ann Thorac Surg. 2018 Mar;105(3):763-9. 
91. Schoen FJ, Levy RJ. Calcification of Tissue Heart Valve Substitutes : Progress Toward Understanding and Prevention. 2005;

92. Dove J, Howanec M, Thubrikar M. Carpentier-Edwards ThermaFix Process: a method for extracting calcium binding sites from pericardial tissue. Edwards Lifesciences LLC. 2006;

93. Flameng W, Hermans H, Verbeken E, Meuris B. A randomized assessment of an advanced tissue preservation technology in the juvenile sheep model. J Thorac Cardiovasc Surg . 2015;149(1):340-5.

94. Lifesciences E. INSPIRIS RESILIA Aortic Valve, Model 11500A, DRAFT. 2018;

95. Gabbay S, Wheatley DJ. Advances in Anticalcific and Antidegenerative Treatment of Heart Valve Bioprostheses. Silent Partners, Inc.; 1997. 105-113 p.

96. McGonagle-Wolff K, Schoen FJ. Morphologic findings in explanted mitroflow pericardial bioprosthetic valves. Am J Cardiol. 1992 Jul;70(2):263-4.

97. Ruzicka DJ, Hettich I, Hutter A, Bleiziffer S, Badiu CC, Bauernschmitt R, et al. The Complete Supraannular Concept: In Vivo Hemodynamics of Bovine and Porcine Aortic Bioprostheses. Circulation. 2009 Sep 15;120(11_suppl_1):S139-45.

98. Meuris B, De Praetere H, Strasly M, Trabucco P, Lai JC, Verbrugghe P, et al. A novel tissue treatment to reduce mineralization of bovine pericardial heart valves. J Thorac Cardiovasc Surg. 2018;156(1):197-206.

\section{Table Legend}

Table 1 - Comparion of the chemical, mechanical and immunological advances of bioprosthetic valves. R: references.

\section{Hosted file}

Table 1.docx available at https://authorea.com/users/360277/articles/481946-bioprostheticaortic-valve-degeneration-a-review-from-a-basic-science-perspective

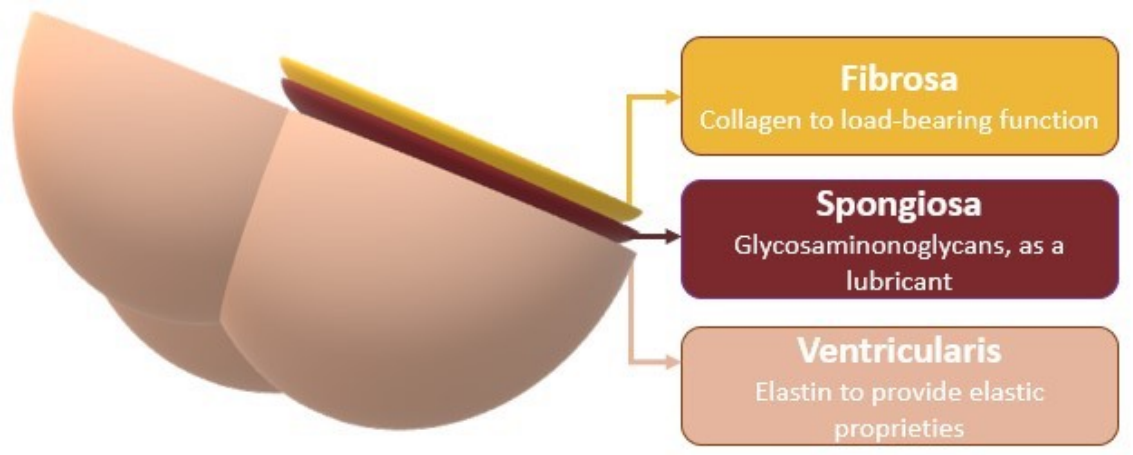




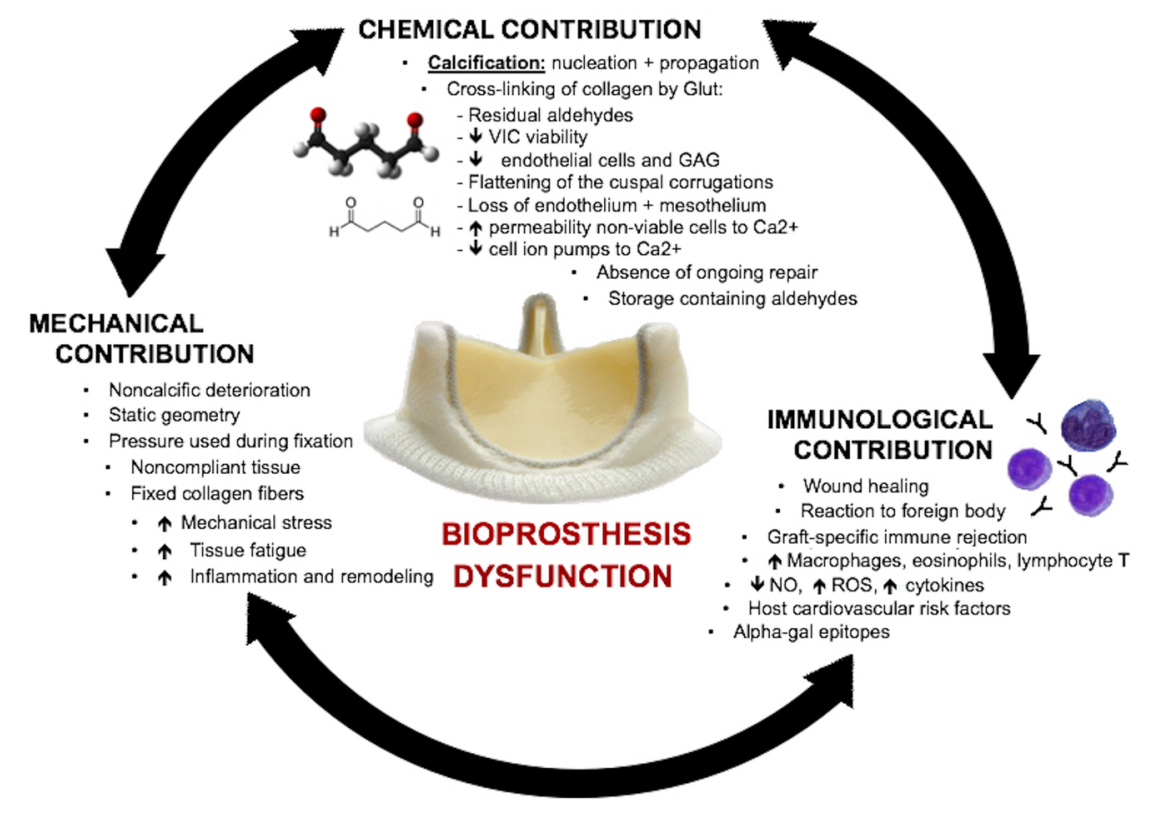

